\title{
Clinical and Histological Features in Wegener Granulomatosis
}

\author{
Li Cavoli Gioacchino ${ }^{1, *}$, Passantino Rita ${ }^{2}$, Bono Luisa ${ }^{1}$, Tortorici Calogera ${ }^{1}$, Ferrantelli Angelo ${ }^{1}$, Giammarresi \\ Carlo ${ }^{1}$, Rotolo Ugo ${ }^{1}$ \\ ${ }^{1}$ Nephrology and Dialysis, Civic and Di Cristina Hospital, Palermo, Italy \\ ${ }^{2}$ Anatomic Pathology, Civic and Di Cristina Hospital, Palermo, Italy \\ *Corresponding author: gioacchinolicavoli@libero.it
}

Received December 24, 2012; Revised January 19, 2013; Accepted February 15, 2013

\begin{abstract}
Wegener Granulomatosis is a systemic ANCA-associated Vasculitis, affecting small-to-medium vessels. Clinical presentation with simultaneous involvement of kidney and upper and lower respiratory tract is unusual. There are few histological reports regarding lung biopsy in WG because clinical and radiologic features are diagnostic. Sometimes radiologic findings can be unusual. We detected an instructive case of WG, analyzing clinical course, laboratory and radiological features, kidney, lung and larynx histological pictures. Besides renal biopsy, nephrology team performed larynx and lung biopsies because of unusual clinical presentation, computed tomography chest examination and relevant malignancy risk regarding following immunosuppressant therapy. WG is a point of interest for clinical multidisciplinary activity. Diagnosis of WG is a combination of clinical, laboratory and pathologic features. Collaboration between different specialities help to improve current diagnostic activity.
\end{abstract}

Keywords: Vasculitis, ANCA, Renal failure

\section{Introduction}

Wegener Granulomatosis (WG) is a systemic antineutrophil cytoplasmic antibodies (ANCA)-associated Vasculitis (AAV), affecting small-to-medium vessels. WG usually starts as a granulomatous disease of respiratory tract and progresses to systemic vasculitis, suggesting an aberrant cell-mediated immune response to exogenous or endogenous antigens, resulting in granuloma formation. In WG, granulomas may represent lymphoid structures ultimately responsible for ANCA production. The clinical presentation with simultaneous involvement of kidney and upper and lower respiratory tract is unusual. We investigated our recent activity in this topic analyzing radiologic, laboratory and histological features.

\section{Case Presentation}

A 55-year-old man, bricklayer and chain smoker, was admitted for rapidly progressive renal failure and anaemia (Creatinine: $9.8 \mathrm{mg} / \mathrm{dl}$, Hb: 6.8gr/dl)). For 3 months he was suffering from dysphonic voice and dry cough, loss of weight and asthenia, oliguria and oedema. Coagulation, liver function tests and virological markers were unremarkable. Immunological tests were normal except for ANCA test positive with a perinuclear florescence pattern. Computed Tomography (CT) chest examination showed bilateral multiple nodules, like septic or metastatic dissemination, without other typical radiologic features (Figure 1). Bronchoscopy was negative except for a larynx polyp; Renal histology showed extracapillar glomerulonephritis (Figure 2). Lung biopsy displayed a liquefactive necrosis (Figure 3). Larynx histology (Figure 4) showed a (Figure 4) Diagnosis of WG was made. The patient was successfully treated with haemodialysis, haemotransfusions, steroid and cyclophosphamide therapy with overall improvement and partial recovery of renal function. He was discharged with a once-weekly schedule of haemodialytic treatment.

\section{Discussion}

According to Chapel Hill Consensus [1] systemic ANCA-associated vasculitides (AAVs) include ChurgStrauss Syndrome, WG, microscopic polyangiitis (MPA) and its renal limited form, the idiopathic necrotizing crescentic glomerulonephritis. The aetiology of AAVs is still unknown. Genetic factors have been suggested but are not particularly strong [2]. Some Authors proposed that infections with gram-negative bacteria may lead, in a susceptible individual, to the development of AAVs [3]. In vitro and in vivo studies strongly support a pathogenic role for ANCA in AAVs. The adaptive immune response manifested by ANCA interacts with innate immunity, in particular neutrophils and the complement system. Together they target the endothelium, resulting in necrotizing vasculitis [4]. In WG and MPA, necrotizing glomerulonephritis and necrotizing pulmonary capillaritis may well result from an injury orchestrated by ANCA. Untreated WG and MPA normally are rapidly progressive and fatal. Pulmonary capillaritis with alveolar haemorrhage is a severe complication in patients with MPA and WG. The most common initial features are respiratory tract symptoms [5]. The initial and contemporaneous presentation with upper and lower respiratory tract and nephropathy is unusual. In this 
patient we observed a clinical starting with laryngeal polyp, bilateral lung involvement and progressive renal failure. A wide spectrum of radiographic abnormalities characterizes WG. The classical radiological finding in necrotizing granulomatosis is cavitated nodules but airspace consolidations and ground-glass opacities may occur with or without presence of nodules [6]. Other less common pulmonary abnormalities are bronchovascular thickening, mild bronchiectasis, segmental atelectasis, pulmonary infarcts, pleural effusion or thickening, and rarely, hilar and Sometimes radiologic presentation can be unusual. In the case shown here we observed bilateral multiple solid nodules without more other frequent findings. There are few lung biopsy reports because clinical and radiologic features are diagnostic. The relevant malignancy risk of this patient (bricklayer and chain smoker subject) leaded nephrology team to perform larynx and lung biopsies before starting immunosuppressive treatment. WG provides an important model for the role of antibodies in mucosal autoimmunity. ANCA are consistently found in 75-95\% of patients with active WG or MPA [6]. The more frequent pattern of ANCA detected in WG is cytoplasmic pattern (c-ANCA with specificity for proteinase 3 ), with a sensitivity of 70$80 \%$ [8]. In this case we detected the less frequent, perinuclear pattern ( $\mathrm{p}$-ANCA with specificity for mieloperoxidase). We think $\mathrm{WG}$ is a point of interest for laboratory, pathology, ORL, nephrology and pneumology team. Variable clinical presentation of AAVs needs of interdisciplinary evaluation. Diagnosis of $\mathrm{WG}$ is a combination of clinical, laboratory and pathologic features. Multidisciplinary collaboration allows to improve diagnostic activity.

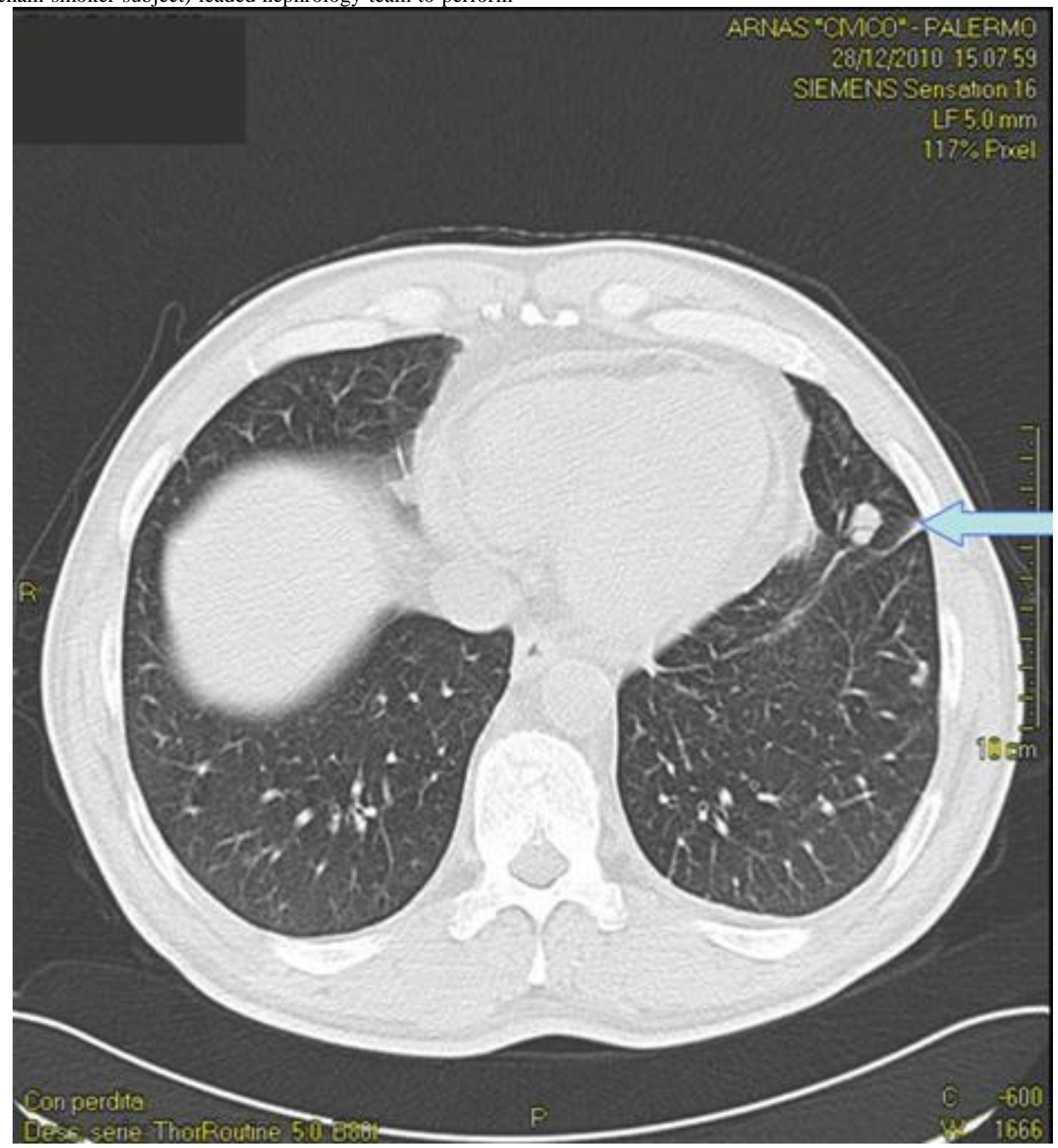

Figure 1. Lung TC scan: Bilateral multiple nodules; arrow : lingula, nodule biopsied. 


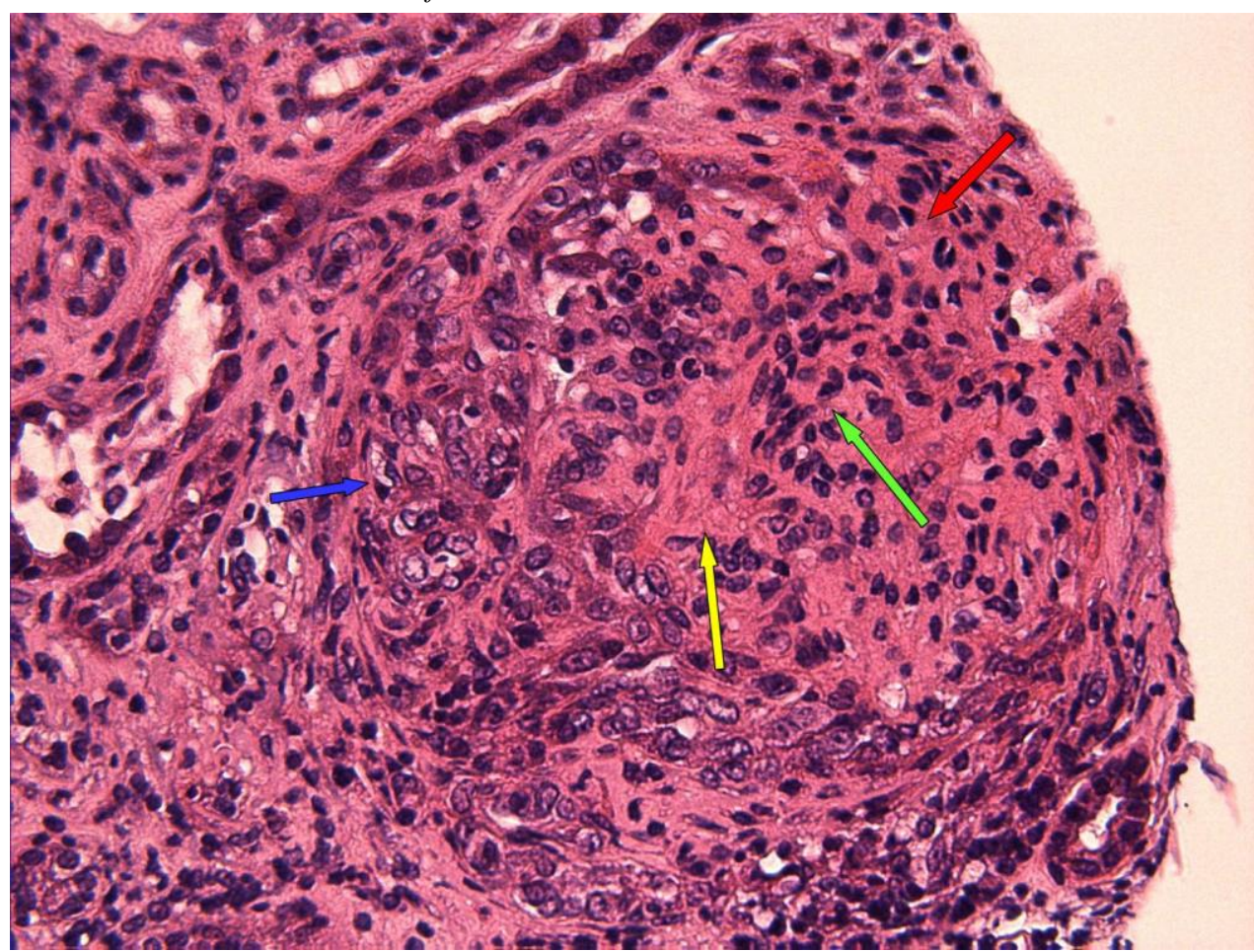

Figure 2. Renal biopsy: Crescentic glomerulonephritis: wide destruction of Bowman's capsule (red arrow), large semicircumferential cellular crescent (blue arrow) with moderate infiltration by neutrophils and predominant mononuclear leukocytes (green arrow), segmental glomerular sclerosis (yellow arrow) and loss of many capillary loops (yellow arrow). ( $\mathrm{H}-\mathrm{E}$ )

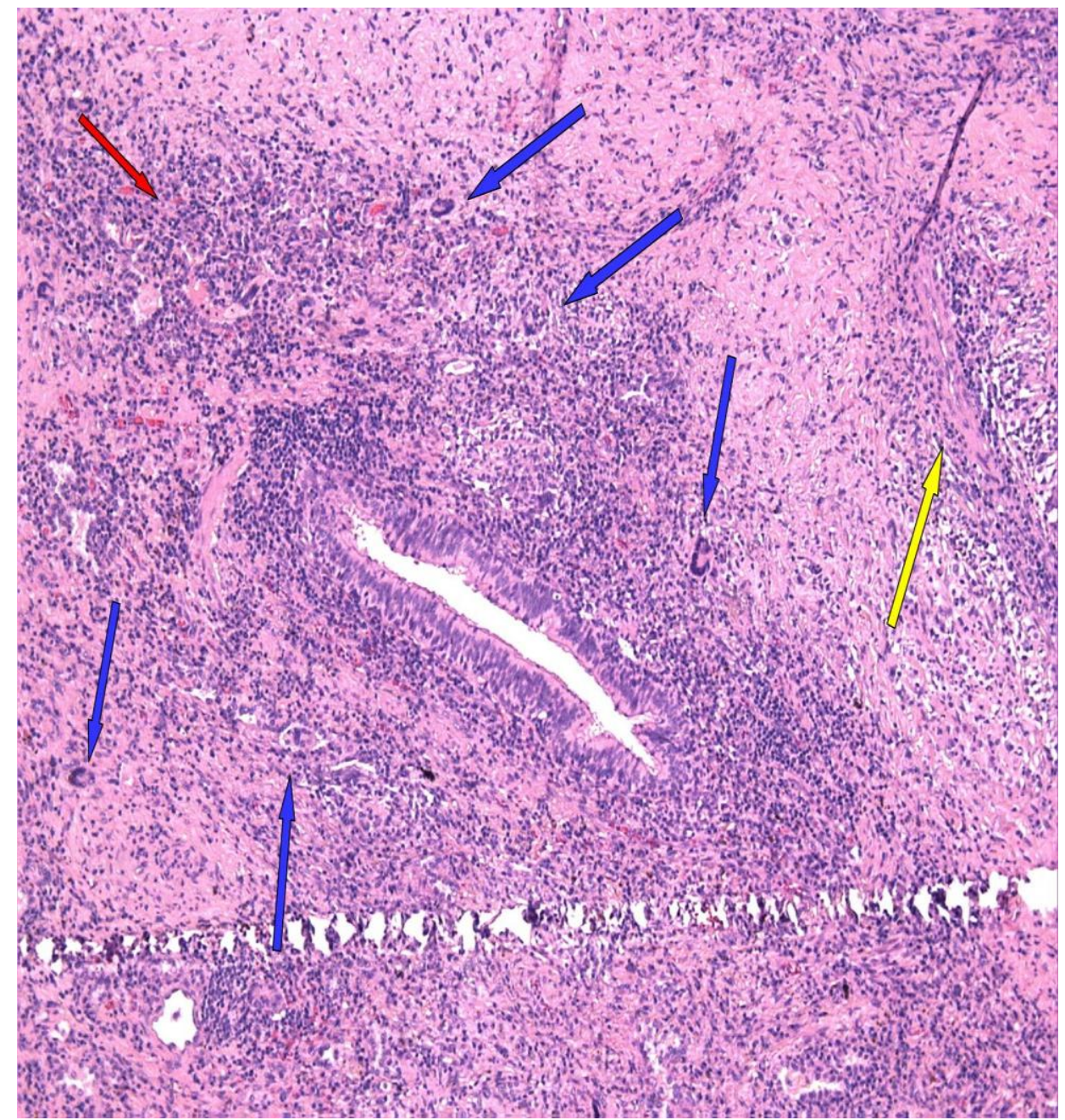

Figure 3. Lung biopsy: liquefactive necrosis, lymphocytes, plasma cells (red arrow) and multinucleated giant cells (blue arrow) that generally do not form well-defined granulomas and a destructive, leukocytolytic angiitis involving arteries and veins (yellow arrow). (H - E). 


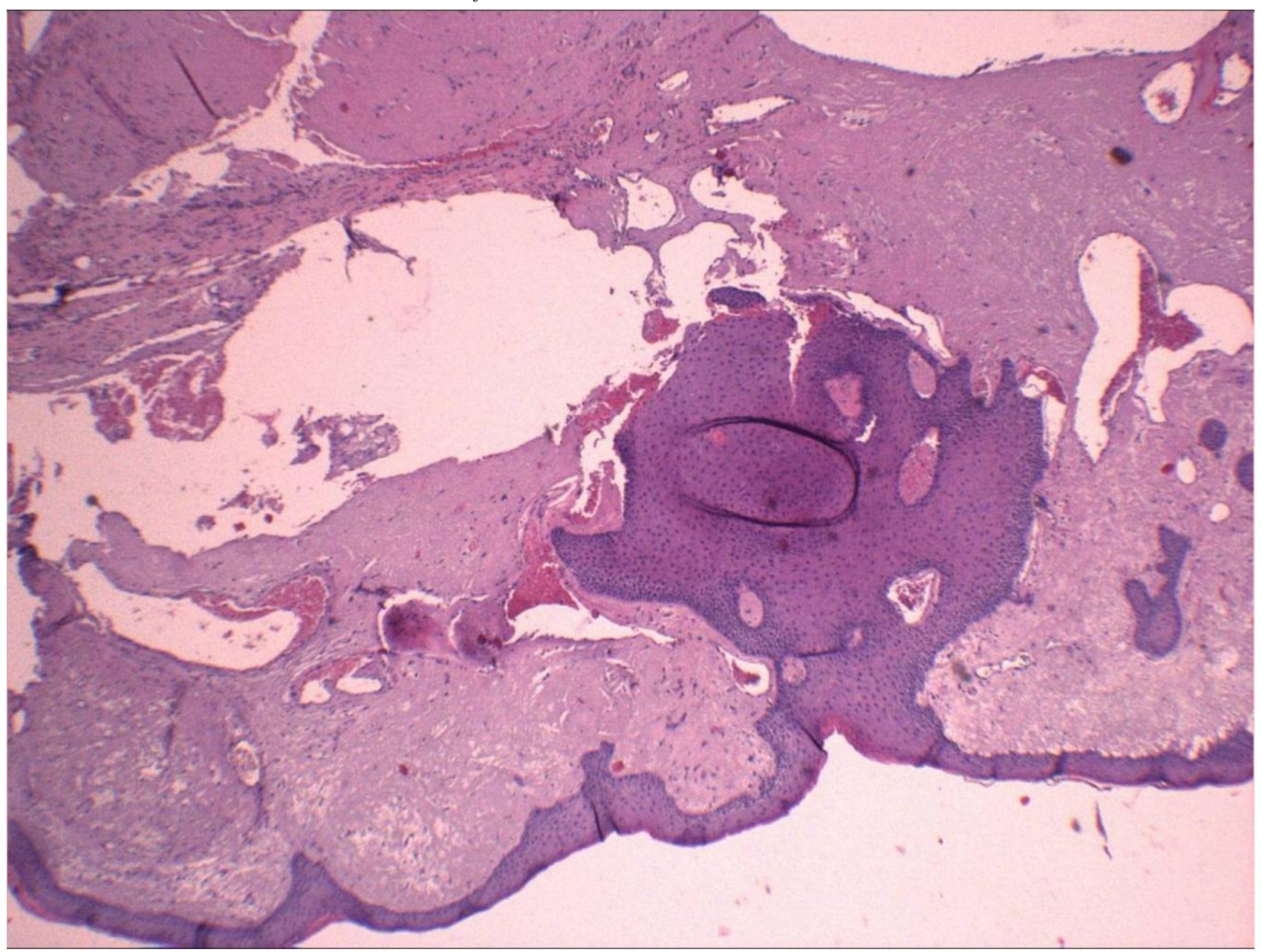

Figure 4. Larynx: nodule ulcerated with angiomatoid features of the stroma, severe necrotizing chronic active aspecific phlogosis without multinucleated giant cells or haemorrhage ( H-E ).

\section{Conflict of interest}

No declared

\section{References}

[1] Jennette JC, Falk RJ, Andrassy K, et al.: Nomenclature of systemic vasculitides: Proposal of an international consensus conference. Arthritis Rheum 1994; 37: 187-92.

[2] Wieczorek S, Holle JU, Epplen JT. Recent progress in the genetics of Wegener's granulomatosis and Churg-Strauss syndrome. Curr Opin Rheumatol. 2010; 22:8-14.
[3] Kain R, Exner M, Brandes R, et al. Molecular mimicry in pauciimmune focal necrotizing glomerulonephritis. Nat Med. 2008;14:1088-1096.

[4] Kallenberg CG. Pathophysiology of ANCA-associated small vessel vasculitis. Curr Rheumatol Rep. 2010 Dec;12:399-405.

[5] Ibrahim S. Focal segmental glomerulosclerosis as the sole renal lesion in Wegener's granulomatosis. Saudi J Kidney Dis Transpl. 2010 Nov; 21:1118-21.

[6] Halshtok O, Eshet Y. Computed tomography scan in necrotizing granulomatosis. Isr Med Assoc J. 2010; 12: 191.

[7] Sinico RA, Di Toma L, Maggiore U, Bottero P, Radice A, Tosoni $\mathrm{C}$ et al. Prevalence and clinical significance of antineutrophil cytoplasmic antibodies in Churg-Strauss syndrome. Arthritis Rheum. 2005; 52: 2926-35.

[8] Csernok E, Holle J, Hellmich B, Willem J, Tervaert C, Kallenberg $\mathrm{CG}$, et al. Evaluation of capture ELISA for detection of antineutrophil cytoplasmic antibodies directed against proteinase 3 in Wegener's granulomatosis: first results from a multicentre study. Rheumatology 2004; 43: 174-80. 